\title{
A New Method for the Determination of Sucrose Concentration in a Pure and Impure System: Spectrophotometric Method
}

\author{
A. Borji, Fz. Borji, and A. Jourani \\ Laboratoire PCPM, Faculté des Sciences et Techniques, Université Hassan 1er, BP 557, Settat, Morocco \\ Correspondence should be addressed to A. Borji; a.borji@uhp.ac.ma
}

Received 8 August 2017; Accepted 14 November 2017; Published 28 December 2017

Academic Editor: Adil Denizli

Copyright (C) 2017 A. Borji et al. This is an open access article distributed under the Creative Commons Attribution License, which permits unrestricted use, distribution, and reproduction in any medium, provided the original work is properly cited.

\begin{abstract}
Analytical chemistry is a set of procedures and techniques used to identify and quantify the composition of a sample of material. It is also focused on improvements in experimental design and the creation of new measurement tools. Analytical chemistry has broad applications to forensics, medicine, science, and engineering. The objective of this study is to develop a new method of sucrose dosage using a spectrophotometry method in a pure and impure system (presence of glucose and fructose). The work performed shows the reliability of this method. A model linking sucrose solution absorbance and mass percentage of glucose and fructose has been developed using experimental design. The results obtained show that all the investigated factors (sucrose concentration, mass percentage of glucose, and mass percentage of fructose) have a positive effect on the absorbance. The effect of the interaction between glucose and fructose on the absorbance is very significant.
\end{abstract}

\section{Introduction}

Analytical chemistry is a measurement science consisting of a set of powerful ideas and methods that are useful in all fields of science and medicine. It is applied throughout industry, medicine, and all the sciences [1].

Dosage has a primary role in the field of chemical analysis, allowing the user of a product or a substance quick and precise answers to any question related to its characterization.

Sucrose and other carbohydrates can be easily distinguished, either by taste or by means of easily developed physical and chemical reactions. The dosage of the latter can be achieved by several methods, either by physical, chemical, or biological methods using enzymes $[2,3]$.

Dosage by physical methods is used to dose sucrose in technical or purified sugar solutions, especially those which are marketed under the name of "liquid sugars". These techniques will mainly be used for high-sucrose processed products [4]. The best known methods are polarimetry [5-7], refractometry $[8,9]$, and those using densimeter.

In spite of their high degree of accuracy, physical methods can only be used for the titration of pure sucrose solutions. If solutions are more complex, with the presence of reducing sugars, colorants, or flavor enhancers, chemical methods can be employed to determine sucrose content. The most widely used chemical methods are chromatography $[10,11]$ and reducing sugars, which are based on the reducing properties of free carbonyl group in reducing sugars, reacted with a copper solution in alkaline medium [12].

As for biological methods, their principle is extensively described in the specialized literature, especially in Bergmeyer's work [13].

The objective of this paper is to develop a new method for the determination of sucrose by spectrophotometer. The influence of certain impurities, such as glucose and fructose, on the sucrose dosage, using this method, has been studied.

\section{Materials and Methods}

2.1. Reagents. The reagents used in this study are

(i) sucrose;

(ii) glucose;

(iii) fructose.

The reagents employed are "of analytical" quality to avoid any other impurities which may influence the measurements. 


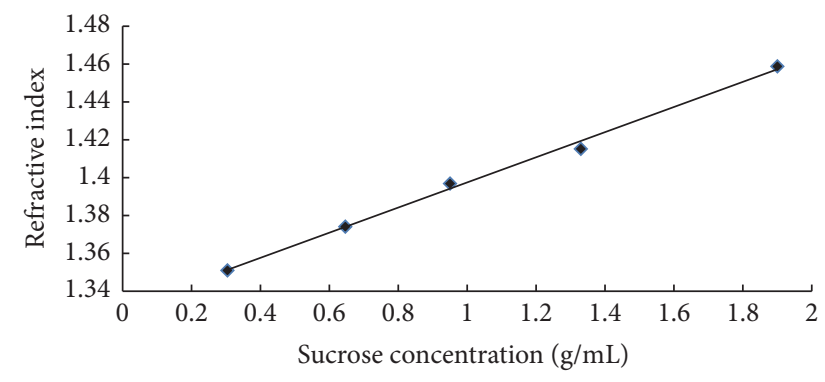

FIGURE 1: Calibration curve for the refractometer.

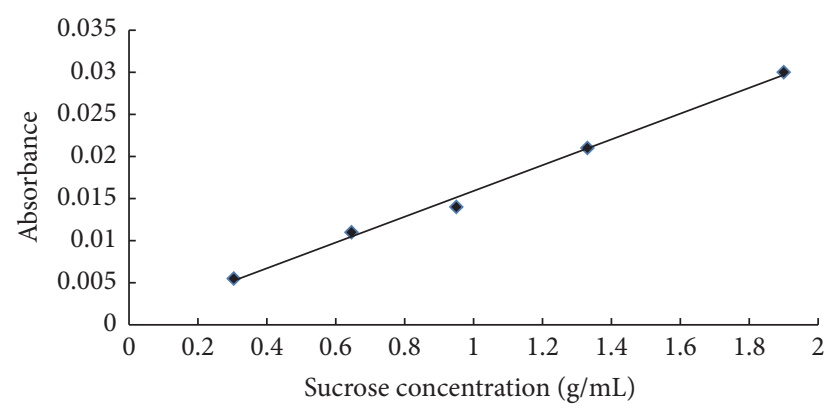

FIgURE 2: Calibration curve for the spectrophotometer.

2.2. Preparation of Samples. The solutions prepared for sucrose dosage are

(i) stock solution of $1.9 \mathrm{mg} / \mathrm{mL}$ sucrose;

(ii) diluted solutions of concentration: respectively, 0.304, $0.646,0.95,1.33$, and $1.9 \mathrm{~g} / \mathrm{mL}$ of sucrose.

2.3. Experimental Protocol. The absorbance of all diluted samples from the stock solution was measured using a spectrophotometer at a wavelength of $420 \mu \mathrm{m}$. The measurements of the refractive index have been performed in order to compare between spectrophotometric and refractometric methods.

\section{Experimental Results}

3.1. Dosage of Sucrose. Among the most frequently used methods for sucrose dosage in aqueous solutions, we find the refractometric method. The bijective relation between the refractive index of pure sucrose solution and its concentration can be used to titrate sucrose in aqueous solutions [14]. For this reason it was chosen as a tool for comparison with the spectrophotometric method.

The calibration equations for the refractometer and the spectrophotometer obtained are, respectively, shown in Figures 1 and 2.

These results indicate that there is good correlation between sucrose concentration and absorbance on the one hand and refractive index on the other, whose calibration equations are $\mathrm{Ab}=0.0153 \mathrm{C}+0.0006$ for the spectrophotometer, with a correlation coefficient of $99.5 \%$, and $n=0.0664 C+1.3311$ for the refractometer, with a correlation coefficient of $99.6 \%$, where $\mathrm{Ab}, n$, and $C$ are the absorbance,
TABLE 1: Validation of the calibration equation for the spectrophotometric method.

\begin{tabular}{ll}
\hline Concentration $(\mathrm{g} / \mathrm{mL})$ & 0.418 \\
Absorbance & 0.008 \\
Calculated concentration & 0.438 \\
Absolute error & 0.020 \\
\hline
\end{tabular}

TABLE 2: Validation of the calibration equation for the refractometer.

\begin{tabular}{ll}
\hline Concentration $(\mathrm{g} / \mathrm{mL})$ & 1.267 \\
Refractive index & 1.416 \\
Calculated concentration & 1.279 \\
Absolute error & 0.012 \\
\hline
\end{tabular}

the refractive index, and the sucrose concentration, respectively.

Repeated tests (4 times) confirm the reproducibility of these results, with the standard deviations being $10^{-4}$ and $8.33 \times 10^{-4}$ for spectrophotometer and refractometer, respectively.

In order to test these two methods, the absorbance and the refractive index of known concentrations of sucrose solutions were measured. The results obtained have been compared with those calculated by the calibration equations (see Tables 1 and 2).

These results show the reliability of sucrose dosage by spectrophotometric method. It should be noted that the refractometric method is more sensitive than the latter. The sensitivity of these two methods, with respect to glucose and fructose, has been studied.

3.2. Influence of Glucose. After the sucrose dosage in aqueous solutions by spectrophotometric method has been validated, the influence of glucose on the absorbance and on the refractive index has been studied. Figure 3 shows the variation of the absorbance and the refractive index as a function of the mass percentage of glucose and for a sucrose concentration of $0.5 \mathrm{~g} / \mathrm{mL}$.

From these results (Figure 3), it can be seen that the effect of glucose starts from $1.56 \%$ for the refractometer and from $1.75 \%$ for the spectrophotometer. Therefore, the latter can be used to titrate sucrose solutions in impure system (presence of glucose with a percentage which does not exceed $1.75 \%$ ). In the following, the absorbance will be modeled as a function of the sucrose concentration and the mass percentages of glucose and fructose, using the experimental design, with the aim of developing a tool capable of measuring the sucrose concentration in impure system (presence of glucose and fructose).

3.3. Modeling of Sucrose Solution Absorbance. Effect of glucose and fructose on the sucrose solution absorbance was studied, using the experimental design. A model linking the sucrose solution absorbance and these two monosaccharides has been established. 
TABLE 3: The experimental ranges and levels of independent variables.

\begin{tabular}{|c|c|c|c|c|}
\hline Factors & Symbol & Low level $(-1)$ & High level $(+1)$ & Unit \\
\hline Concentration of sucrose & $x_{1}$ & 0.3 & 1.1 & $\mathrm{~g} / \mathrm{mL}$ \\
\hline Mass percent of glucose & $x_{2}$ & 0.1 & 4 & $\%$ weight \\
\hline Mass percent of fructose & $x_{3}$ & 0.1 & 4 & $\%$ weight \\
\hline
\end{tabular}

TABLE 4: Experimental design matrix.

\begin{tabular}{llccrr}
\hline Experiment & $I$ & $x_{1}$ & $x_{2}$ & $x_{3}$ & -1 \\
\hline$(1)$ & 1 & -1 & -1 & -1 & 0.013 \\
$(2)$ & 1 & 1 & -1 & -1 & 0.030 \\
$(3)$ & 1 & 1 & 1 & 1 & 0.031 \\
$(4)$ & 1 & -1 & -1 & 1 & 0.014 \\
$(5)$ & 1 & 1 & 1 & -1 & 0.038 \\
$(6)$ & 1 & -1 & 1 & 1 & 0.015 \\
$(7)$ & 1 & -1 & -1 & 0.026 \\
$(8)$ & 1 & 1 & & 1 & 0.033 \\
\hline
\end{tabular}

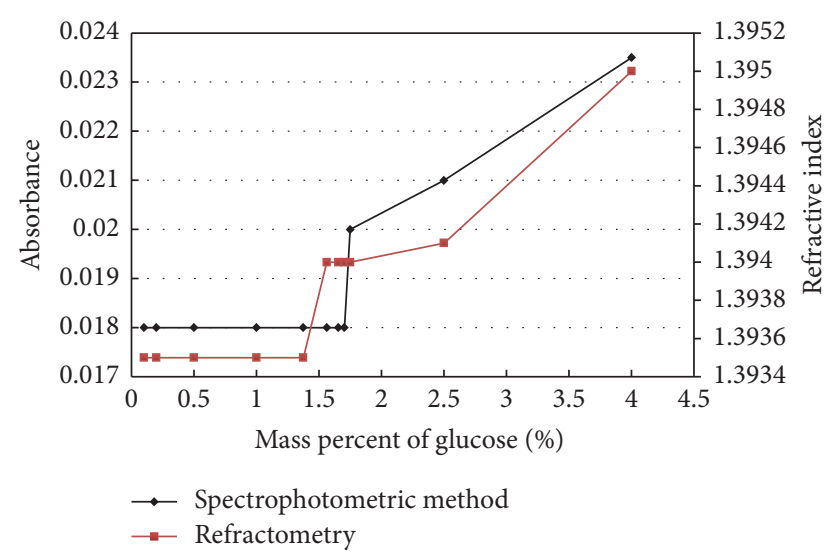

FiguRE 3: Influence of glucose on absorbance and refractive index.

The technique of statistical design for the experiments can be used for process characterization, optimization, and modeling.

Basically, the classical parameter design is complicated and not easy to use; in particular, a large number of experiments must be conducted when the number of the process parameters increased. For this reason, the design of experiments is a useful tool to study the interactions between two or more variables at reduced number of experimental trials [15]. The factorial designs determine which factors have the important effects on the response and how the effect of one factor varies with the level of the other factors. The effects are the differential quantities expressing how a response changes as the levels of one or more factors are changed. Also, factorial designs allow measuring the interaction between each different group of factors [16].

If we call $n$ the number of variables to be tested, in order to measure the effect of all the variables combinations when each variable is tested at a high and a low level, $2^{n}$ experiments will be needed [17]. In this study, three factors were chosen as independent variables, namely, concentration of sucrose $\left(x_{1}\right)$, mass percent of glucose $\left(x_{2}\right)$, and mass percent of fructose $\left(x_{3}\right)$.

The natural values of each factor and their respective levels are presented in Table 3. The selection of levels of different factors is carried out on the basis of the preliminary trials: sucrose concentration ranging from 0.3 to $1.1 \mathrm{~g} / \mathrm{mL}$, mass percent of glucose from 0.1 to $4 \%$ weight, and mass percent of fructose from 0.1 to $4 \%$ weight. The design performed according to Table 4 was composed of $2^{3}$ factorial designs.

Table 4 shows the results of the 8 tests carried out.

The coded values of $x_{j}$ were obtained from the following relationship [18-20]:

$$
x_{j}=\frac{Z_{j}-Z_{j}^{0}}{\Delta Z_{j}} \quad j=1,2, \ldots, k,
$$

with

$$
\begin{aligned}
Z_{j}^{0} & =\frac{Z_{j \max }+Z_{j \min }}{2}, \\
\Delta Z_{j} & =\frac{Z_{j \max }-Z_{j \min }}{2},
\end{aligned}
$$

where $x_{j}$ is the coded value of $j$ th variable, $Z_{j}$ is the encoded value of $j$ th variable, $Z_{j}^{0}$ is the value of $Z_{j}$ at the center point of the investigation domain, and $\Delta Z_{j}$ is the step size. Here, $Z_{j \max }$ and $Z_{j \min }$ represent the maximum and the minimum level of factor $j$ in natural unit, respectively. The experimental data are analyzed by full factorial design to fit the following first-order polynomial equation [21-23]:

$$
\begin{aligned}
y= & b_{0}+\varepsilon+b_{1} x_{1}+b_{2} x_{2}+b_{3} x_{3}+b_{12} x_{1} x_{2}+b_{13} x_{1} x_{3} \\
& +b_{23} x_{2} x_{3}+b_{123} x_{1} x_{2} x_{3},
\end{aligned}
$$

where $y$ the estimated sucrose solution absorbance; $b_{0}$ is the value of fitted response at the center point of design; $b_{j}$ and $b_{j i}$ are the linear and interaction terms, respectively $[24,25]$. 
TABLE 5: Comparison between the measured absorbance and the calculated absorbance.

\begin{tabular}{lccc}
\hline Test & Calculated absorbance & Measured absorbance & Absolute error \\
\hline$(0,0,0)$ & 0.0250 & 0.026 & 0.001 \\
$(0.5,0.5,0.5)$ & 0.0317 & 0.032 & 0.0003438 \\
$(-0.5,-0.5,-0.5)$ & 0.0186 & 0.019 & 0.0004063 \\
\hline
\end{tabular}

When the response data are obtained from the test work, a regression analysis is carried out to determine the coefficients of the response model $\left(b_{1} ; b_{2} ; \ldots ; b_{n}\right)$, as well as their standard errors and their significance. In addition to the constant $\left(b_{0}\right)$ and error $(\varepsilon)$ terms, the response model incorporates [26]

(i) linear terms in each of the variables $\left(x_{1}, x_{2}, \ldots, x_{n}\right)$;

(ii) first-order interaction terms for each paired combination $\left(x_{1} x_{2}, x_{1} x_{2}, \ldots, x_{n-i} x_{n}\right)$.

In general (3) can be written in matrix form:

$$
Y=B X+\varepsilon
$$

The $B$ coefficients, which should be determined in the second-order model, are obtained by Goupy [21]:

$$
B=\left[X^{T} \cdot X\right]^{-1} \cdot[X]^{T} \cdot Y,
$$

where $B$ is the column matrix of estimated coefficients; $\left[X^{T} \cdot X\right]^{-1}$ the dispersion matrix; $[X]^{T}$ the transpose matrix of experiments matrix $[X]$ and $Y$ is the column matrix of observations.

The model equation for absorbance of sucrose solution was obtained after performing eight experiments and discarding the insignificant effect $\left(b_{13}\right.$ and $\left.b_{123}\right)$ :

$$
\begin{aligned}
\widehat{y}= & 0.02500+0.008000 x_{1}+0.002500 x_{2} \\
& +0.002750 x_{3}-0.001000 x_{1} x_{2}+0.001750 x_{2} x_{3} .
\end{aligned}
$$

The model's coefficients were estimated using Minitab software.

In order to validate this model, it is tested using the center, $1 / 4$, and $3 / 4$ of the range of each factor. The results obtained are summarized in Table 5.

According to this test, we find that the empirical model gives results closer to reality, so it can be used to measure sucrose, either in pure or in impure system (presence of glucose and fructose). The difference between the measured and the predicted values does not exceed $0.1 \%$.

The values obtained by the model ( $\hat{y}$ predicted) are compared with those of experimental data ( $y$ experimental) (Table 6).

A good adjustment of (6) to the experimental data was verified through the high correlation coefficient value obtained: $R^{2}=99.24 \%$ (Figure 4 ). The random distribution of the residuals (Figure 5) shows the absence of a trend, indicating that the mathematical model is adequate and that there is no inconsistency between the experimental and calculated values of the response.

Figures 6 and 7 illustrate the effects of each factor and their interactions on the sucrose solutions absorbance.
TABLE 6: Comparison between observed and predicted responses.

\begin{tabular}{lccc}
\hline Runs & $y$ & $\hat{y}$ & Residuals \\
\hline$(1)$ & 0.013 & 0.0125 & 0.0005 \\
$(2)$ & 0.03 & 0.0305 & -0.0005 \\
$(3)$ & 0.031 & 0.03 & 0.001 \\
$(4)$ & 0.014 & 0.0145 & -0.0005 \\
$(5)$ & 0.038 & 0.039 & -0.001 \\
$(6)$ & 0.015 & 0.016 & -0.001 \\
$(7)$ & 0.026 & 0.025 & 0.001 \\
$(8)$ & 0.033 & 0.0325 & 0.0005 \\
\hline
\end{tabular}

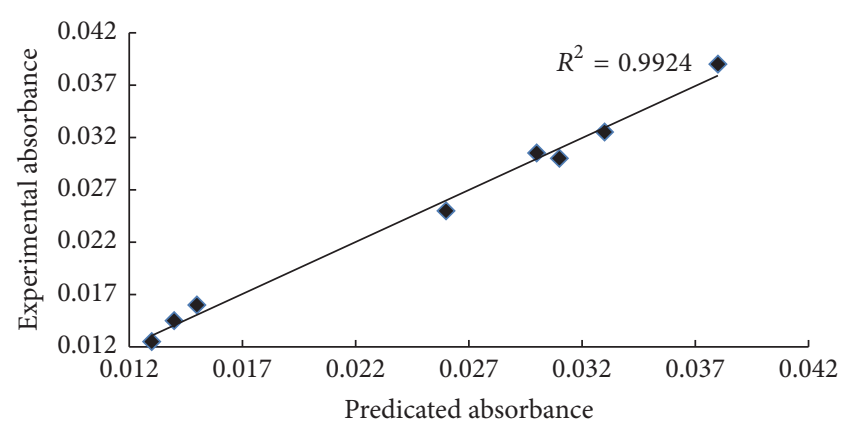

FIGURE 4: Comparison of experimental and predicted responses.

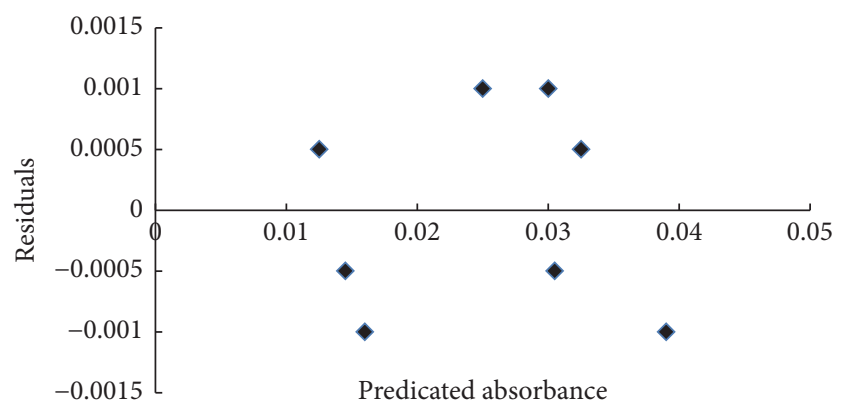

FIGURE 5: Residual analysis for estimated model.

The results show that increasing the percentage of glucose and fructose causes an absorbance increase. The effects of glucose-sucrose and fructose-sucrose interactions are negligible because the effect of these tow monosaccharides on absorbance does not depend on sucrose concentration. On the contrary, the effect of the interaction between fructose and glucose is very significant. 


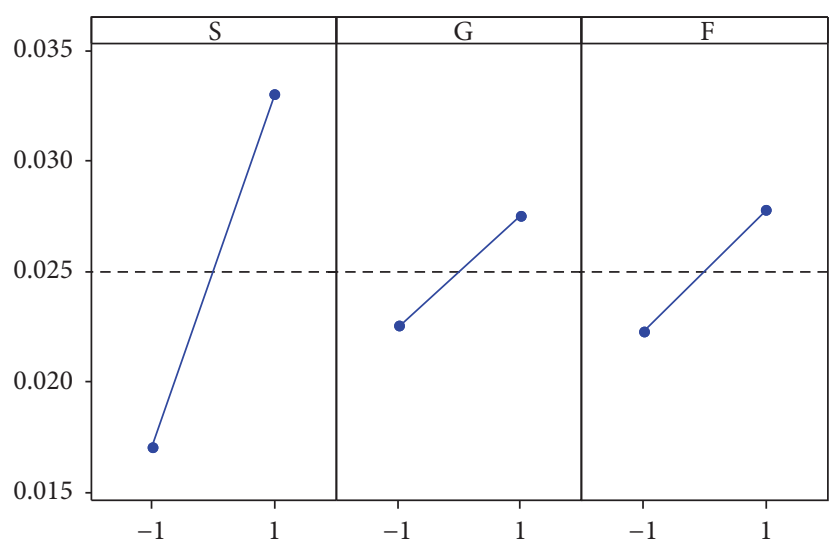

FIGURE 6: Diagram of the main effects for absorbance (S: sucrose; G: glucose; F: fructose).

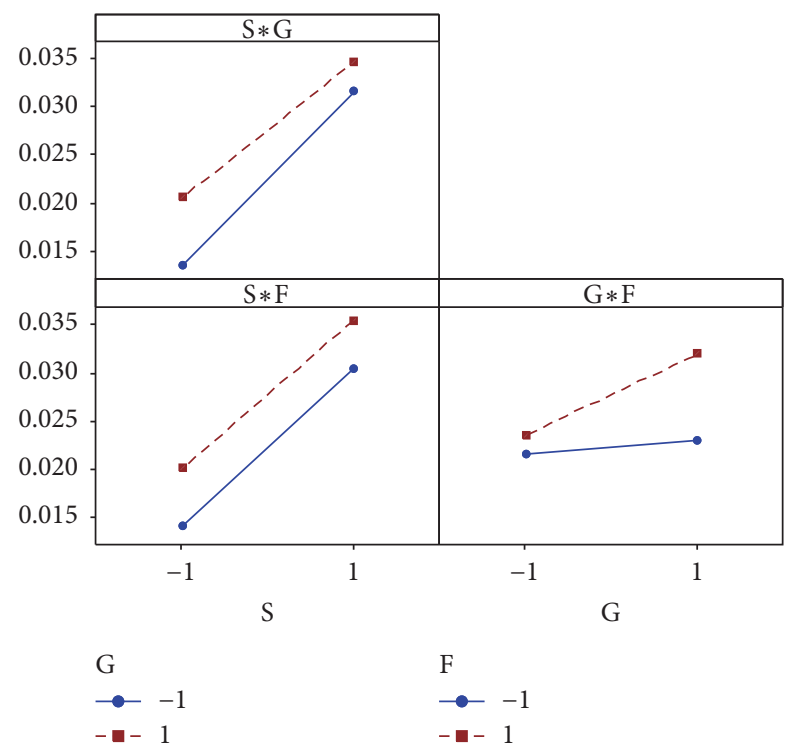

Figure 7: Diagram of interactions for absorbance (S: sucrose; G: glucose; F: fructose).

\section{Conclusion}

In this work, a new spectrophotometric method for the determination of sucrose concentration in a pure and impure system was demonstrated. The calibration equation established was validated and compared with the refractometric method. An empirical model linking the absorbance of sucrose solution and mass percentage of glucose and fructose was developed using the experimental design.

The work performed shows the reliability of this method and that glucose and fructose have a positive effect on the sucrose solution absorbance. Influence of the interaction between these two monosaccharides on absorbance is very significant.

\section{Conflicts of Interest}

The authors declare that there are no conflicts of interest regarding the publication of this paper.

\section{References}

[1] A. E. Kaifer, "Fundamentals of Analytical Chemistry. Sixth edition (Skoog, Douglas A.; West, Donald M.; Hollar, James F.)," Journal of Chemical Education, vol. 69, no. 11, p. A305, 1992.

[2] C. B. Purves and C. S. Hudson, "The analysis of gamma-methylfructoside mixtures by means of invertase. I," Journal of the American Chemical Society, vol. 56, no. 3, pp. 702-707, 1934.

[3] M. Bengtsson and J. Tjebbes, "Automatic enzymatic determination of true sucrosebeet and molasses," Int. Sugar, vol. 93, no. 1110, pp. 121-125, 1991.

[4] M. Mathlouthi and P. Reiser, Sucrose, Springer US, Boston, MA, 1995.

[5] F. Schneider, Sugar Analysis, ICUMSA Publication, UK, Peterborough, 1979.

[6] J. Dutton, "Double polarimetric method," in In Sugar Analysis (ed. F. Schneider), pp. 30-32, ICUMSA Publication, Peterborough, UK, 1979.

[7] V. Singleton, J. Horn, C. Bucke, and M. Adlard, "A New Polarimetric Method For The Analysis Of Dextran And Sucrose, Journal American Society of Sugar Cane Technologists," Volume, vol. 22, 2002.

[8] E. Landt, "ICUMSA Proceedings (9th Session), ICUMSA Publication," in Proceedings of the ICUMSA Proceedings (9th Session) , ICUMSA Publication, pp. 22-25, Peterborough, 1936.

[9] K. J. Rosenbruch, "ICUMSA Proceedings, (17th Session), ICUMSA Publication," in Proceedings of the ICUMSA Proceedings, (17th Session), ICUMSA Publication, pp. 166-174, Peterborough, UK, 1978.

[10] K. J. Schäffler and P. G. Morel du Boil, "A review of gas chromatography in the South Africa Sugar Industry. Development and application of accurate methods for sugar analysis," Sugar Technol. Rev, vol. 11, no. 2, pp. 95-185, 1984.

[11] J. Karr and L. W. Norman, "The Determination of Sucrose in Concentrated Steffen Filtrate by G.L.C," Journal of Sugarbeet Research, vol. 18, no. 1, pp. 53-59, 1974.

[12] Anon, "ICUMSA Methods Book," Tech. Rep., British Sugar Technical Centre, Colney, Norwich, 1994.

[13] H. U. Bergmeyer, Methods of Enzymatic Analysis, Academic Press New York, USA, 1974.

[14] K. J. Rosenbruch, "Refractice index , subject 12 Referees report," in Proceedings of the ICUMSA Proceedings (19th Session, pp. 199212, Peterborough, UK, 1986.

[15] I. Yahiaoui and F. Aissani-Benissad, "Experimental design for copper cementation process in fixed bed reactor using two-level factorial design," Arabian Journal of Chemistry, vol. 3, no. 3, pp. 187-190, 2010.

[16] A. Bozkir and O. M. Saka, "Formulation and investigation of 5FU nanoparticles with factorial design-based studies," Farmaco, vol. 60, no. 10, pp. 840-846, 2005.

[17] L. C. Morais, O. M. Freitas, E. P. Gonçalves, L. T. Vasconcelos, and C. G. González Beça, "Reactive dyes removal from wastewaters by adsorption on eucalyptus bark: Variables that define the process," Water Research, vol. 33, no. 4, pp. 979-988, 1999.

[18] K. Adinarayana and P. Ellaiah, "Response surface optimization of the critical medium components for the production of alkaline protease by a newly isolated Bacillus sp," Journal of Pharmacy \& Pharmaceutical Sciences, vol. 5, no. 3, pp. 272-278, 2002.

[19] R. Sen and T. Swaminathan, "Response surface modeling and optimization to elucidate and analyze the effects of inoculum 
age and size on surfactin production," Biochemical Engineering Journal, vol. 21, no. 2, pp. 141-148, 2004.

[20] D. C. Montgomery, Design and Analysis of Experiments, John Wiley \& Sons, 8th edition, 2012.

[21] J. L. Goupy, "Methods for experimental design," Data Handling in Science and Technology, vol. volume 12, 1993.

[22] E. Bayraktar, "Response surface optimization of the separation of DL-tryptophan using an emulsion liquid membrane," Process Biochemistry, vol. 37, no. 2, pp. 169-175, 2001.

[23] M. Y. Can, Y. Kaya, and O. F. Algur, "Response surface optimization of the removal of nickel from aqueous solution by cone biomass of Pinus sylvestris," Bioresource Technology, vol. 97, no. 14, pp. 1761-1765, 2006.

[24] G. W. Oehlert, A first course in design and analysis of experiments, University of Minnesota, 2010.

[25] G. E. P. Box, W. G. Hunter, and J. S. Hunter, Statistics for Experimenters, John Wiley \& Sons, New York, NY, USA, 1978.

[26] F. A. Pavan, Y. Gushikem, A. C. Mazzocato, S. L. P. Dias, and E. C. Lima, "Statistical design of experiments as a tool for optimizing the batch conditions to methylene blue biosorption on yellow passion fruit and mandarin peels," Dyes and Pigments, vol. 72, no. 2, pp. 256-266, 2007. 

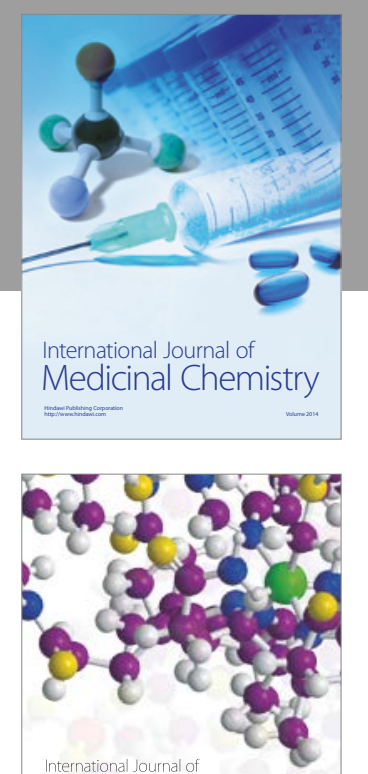

Carbohydrate Chemistry

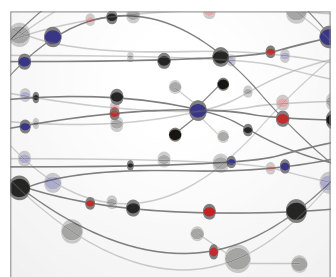

The Scientific World Journal
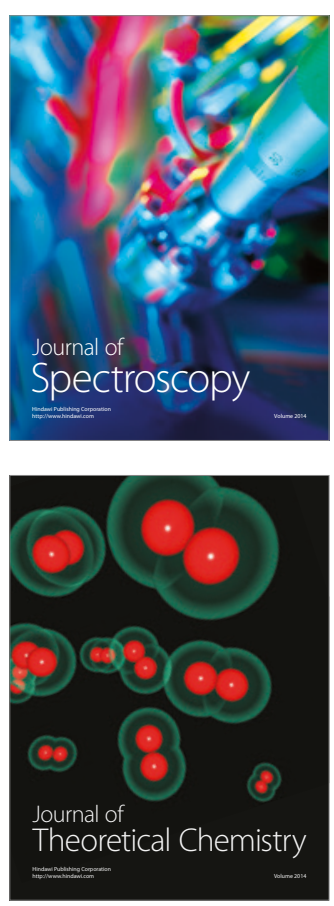
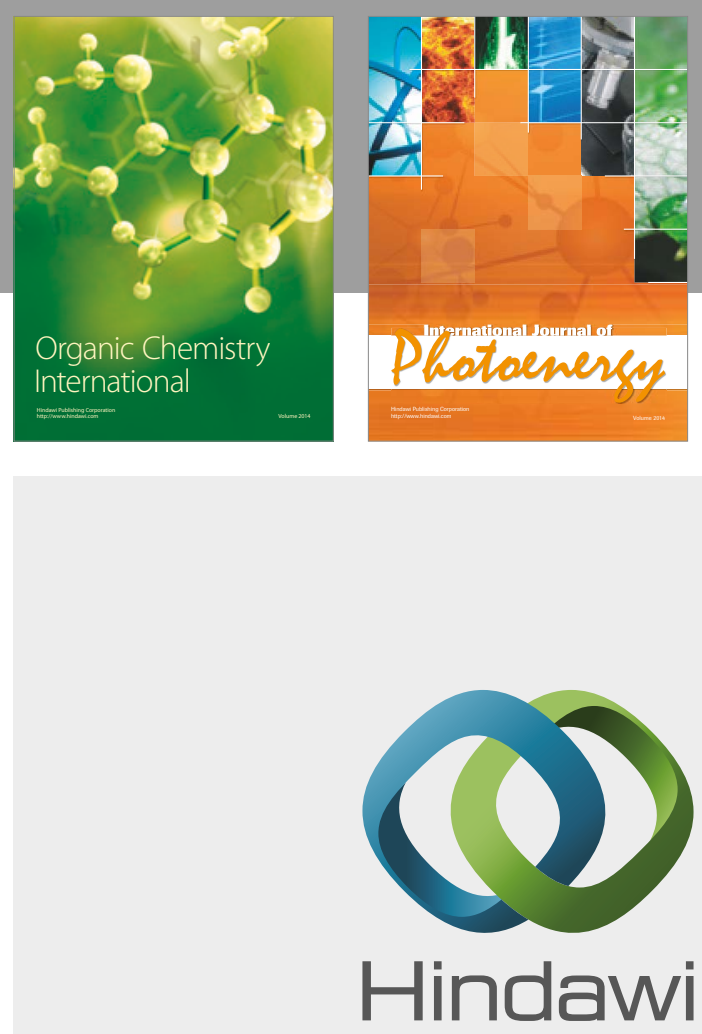

Submit your manuscripts at

https://www.hindawi.com

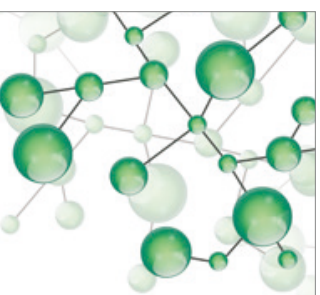

International Journal of

Inorganic Chemistry

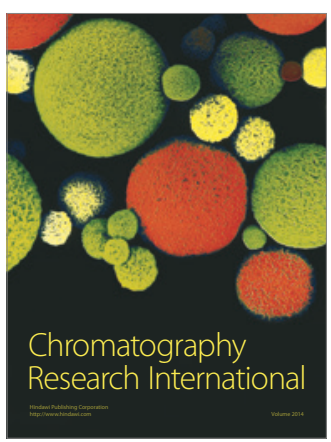

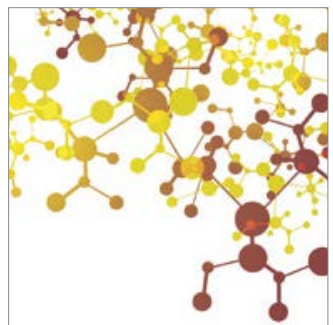

Applied Chemistry
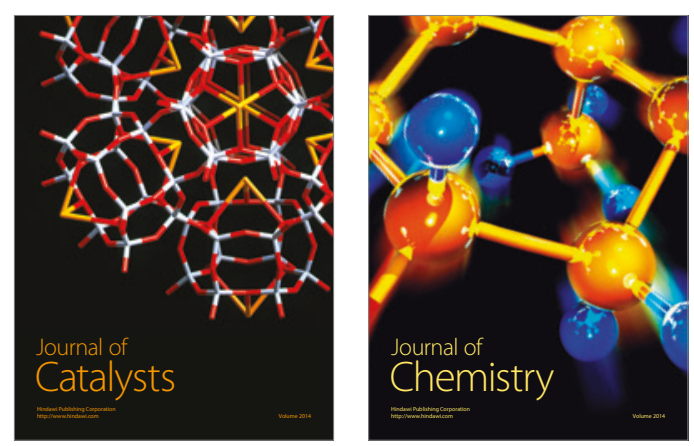
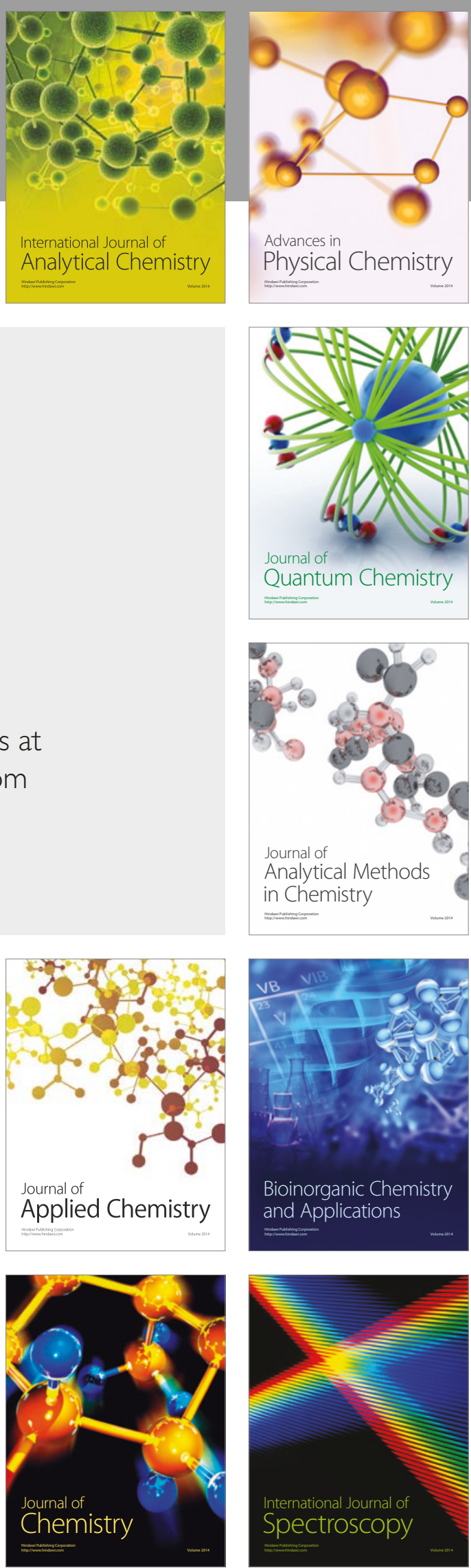